\title{
Performance Evaluation of ENUM Directory Service Design ${ }^{\star}$
}

\author{
Hyewon K. Lee and Youngsong Mun \\ School of Computing, Soongsil University, Seoul, Korea \\ kerenlee@sunny.ssu.ac.kr, mun@computing.ssu.ac.kr
}

\begin{abstract}
ENUM (tElephone NUmbering Mapping) is a protocol that brings convergence between PSTN networks and IP networks using a unique worldwide E.164 telephone number. This mechanism couples two completely different environments with E.164 number and allows users to utilize IP based application services in PSTN networks and PSTN based application services in IP networks. We propose a new way to organize and handle ENUM name servers to improve the performance of name resolution process in ENUM based application service. We have built an ENUM based network model when NAPTR (Naming Authority PoinTeR) resource record is registered and managed by area code at the initial registration step.
\end{abstract}

\section{Introduction}

ENUM maps telephone numbers into domain name and provides a connection between IP network based services and PSTN based services [1]. This mechanism enables endusers to access web-sites, e-mail, fax, mobile-phone or instant message services with a single telephone number. ENUM protocol promises uniqueness, flexibility and convenience to both IP network and PSTN users; however, there is no sufficient proof that the performance of ENUM service is quite good to employ in Internet instead of using conventional DNS. ENUM clients get only specific NAPTR Resource Records (RRs) from name servers, not all NAPTR RRs, and further, NAPTR RRs joined to one E.164 number are too big to be cached in a local name server, so the availability of cached NAPTR RR should be lower than the availability of cached conventional RR in local name servers. Thus, people would have more a relatively long wait to get an appropriate response with ENUM service. In this paper, we discuss lookup service of ENUM, estimate response time and present some techniques to improve its performance.

\section{Related Works}

\subsection{DNS and Name Lookup Service}

IP based applications are indispensable to DNS [2], so the correct operation of DNS plays a prominent part in IP networks. Domain name is preferred to IP address because of its

* This work was done as a part of Information \& Communication fundamental Technology Research Program supported by Ministry of Information \& Communication in republic of Korea. 
readability and writability; however, physical hardware cannot understand this, so DNS translates domain name into the logical IP address of device and provides information to most IP based applications. DNS offers the other service, opposite mapping from IP address to domain name.

DNS makes extensive use of caching to reduce server load, client latency and ultimately DNS traffic on the Internet. It is generally credited that cache is very efficacious even in changeable and variable IP environment because information in DNS database varies slowly, and small quantities of staleness are tolerable. Simply, the DNS caching design prefers availability above freshness [2][6].

\subsection{ENUM Lookup Service}

ENUM first transforms E.164 numbers into ENUM domain names and then uses the DNS-based architecture to access records from which URIs are derived. E.164 numbers is used to provide users with a number of addresses, including those used for phone, fax and email, at which the called party can be contacted.

\section{Design of ENUM Directory Service}

The ENUM delegation model is composed with three tiers: Tier 0 registry, Tier 1 registry, and Tier 2 name server provider. At first, Tier 0 corresponds to the ENUM root level. At this level, the ENUM architecture contains only one domain, called the ENUM root. The Tier 0 name servers contain records that point to ENUM Tier 1 name servers. Secondly, Tier 1 registry is an entity responsible for the whole management of the E.164 country code for ENUM. Name servers of Tier 1 contain records that point authoritative name servers for individual E.164 number or blocks of numbers in the country code. Finally, Tier 2's name servers contain NAPTR RRs with information for specific services. Name servers of Tier 2 responses to queries from other name servers or clients.

Hierarchical design around administratively delegated name spaces and aggressive use of caching are indispensable to the scalability of DNS [2] [6]. Contemporary DNS resolution makes use of caching, and the cache hit rate is up to $90 \%$ depending on the starting state of database, so the response time of DNS query is independent with hop count. On the contrary, the response time of ENUM query is dependent on hop count because a local name server will not cache all of NAPTR RRs with E.164. The ENUM resolution will have excessively low cache hit rate.

The high cache hit rate is closely connected with scalability; so alternatively, we propose to separate Tier 2 name server's role according to local area numbers to improve ENUM performance. If Tier 2 name server represents one or more area codes, each local name server will pre-learn and cache Tier 2 name server's addresses corresponding area codes. More than two Tier 2 name server providers can represent a specific area; and moreover, one Tier 2 name server providers can represent more than one area, but their information should be mutually exclusive except caching. 


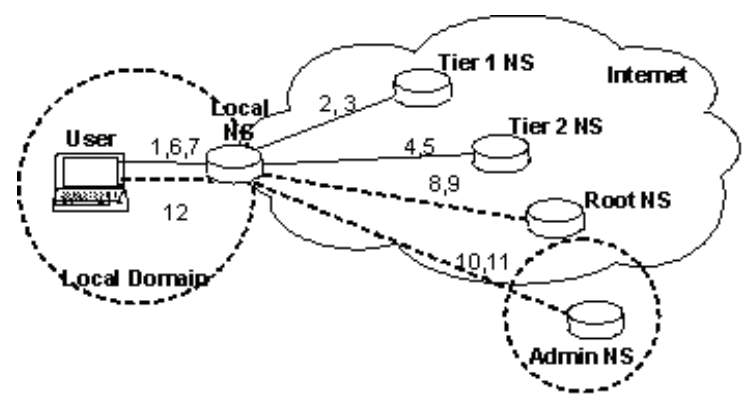

Fig. 1. Schematic topology of traced networks

\section{Performance Evaluation}

\subsection{Modeling}

The base assumptions for this experiment are as follows: domain name to query is restricted only to the $\mathrm{kr}$ domain, so any query whose answer is not in the local name server's zone file or cache, is forwarded to the .kr root name server. A query is only generated in the SSU network, and the local name server is ns.ssu.ac.kr. Name server administrator designates the nearest .kr root name server. The administrative name server does not have any sub name server. Any current DNS name server can be the ENUM Tier 2 name server. Name server processing time for ENUM and DNS lookups is assmed to be same.

Once a local name server receives a query about NAPTR RR from a host, it first searches its cache. If the local name server finds appropriate NAPTR RRs for the query, it responds to the host, and the ENUM resolution is over. Otherwise, the local name server forwards the query to the Tier 1 name server, which then returns a referral response, composed of addresses of administrative Tier 2 name servers. The local name server then forwards the query to the Tier 2 name server again. Then, the Tier 2 name server builds response packet containing NAPTR RRs and send it back to the local name server. As the local name server receives a response, it caches and forwards the response to the host. Now, the ENUM client of the host receives NAPTR RR, and starts DNS name lookup process to map NAPTR RR to IP address. DNS lookup process is identical to ENUM lookup, except that the final response from an administrative name server is IP address, not NAPTR RRs. Fig. 11ilustrates the name lookup process. The solid line indicates the ENUM query process, while the dotted line indicates the conventional DNS query process. The expected response time of each step in name lookup process, $x_{i}$ is as follows, where $i \leq 1$ then $m=i$, else $m=i+1$ :

$$
x_{i}=2 \alpha \sum_{j=1}^{m}\left(n_{j}-1\right)+m \beta+\gamma(i-1)+2 \sum_{j=1}^{m} n_{j} \delta_{j},
$$


where $n_{i} 1$ is a hop count in ith trace, $\alpha$ is router processing time, $\beta$ is DNS name server table searching time, $\gamma$ is DNS name server table caching time, $\delta$ is query propagation delay time between two hops, $p_{\lambda}$ is probability related to ENUM resolution, and $p_{\mu}$ is probability related to original DNS resolution. Especially, $p_{\lambda_{1}}$ is the probability that local name server has NAPTR RR corresponding to a query, $p_{\lambda_{2}}$ is the probability that Tier 1 registry's name server gives address of Tier 2 name server corresponding to a query. Similarly, $p_{\mu_{1}}$ is probability that local name server can response to a query, and $p_{\mu_{2}}$ is the probability that root name server gives the address of administrative name server corresponding to a query.

Assume that $x_{\lambda}$ is the response time of the ENUM resolution process, and $x_{\mu}$ is the response time of the DNS resolution process. The expectation of the response time for the whole resolution process using ENUM is the sum of each expectation.

\subsection{The Data-Statistical Analysis}

To evaluate the response time, the processing speed of root name server, the cache hit rate of local name server, and propagation time between two hops are measured via experiments. For router processing time, simply benchmark from Cisco [4] was used, and the router processing speed will be the reciprocal of throughput.

At first, for name server processing time, 5,000,000 virtual A records were used to get DNS query processing time of .kr root server on July 2003. From Table 1 the processing time of name server is $0.008 \mathrm{~ms}$.

Table 1. Throughput of .kr root name server

\begin{tabular}{|c|c|c|c|}
\hline $\begin{array}{l}\text { DNS program } \\
\text { BIND } 9.2 .0\end{array}$ & $\begin{array}{l}\text { Zone file data } \\
\text { Virtual } 5 * 10^{6} \text { A } \\
\text { records }\end{array}$ & $\begin{array}{l}\text { Query data } \\
\text { Virtual } 5 * 10^{6} \mathrm{~A} \\
\text { records in zone file }\end{array}$ & $\begin{array}{l}\text { Check program } \\
\text { queryperf tool }\end{array}$ \\
\hline $\begin{array}{l}\text { Queries sent } \\
500,000\end{array}$ & $\begin{array}{l}\text { Queries completed } \\
500,000\end{array}$ & $\begin{array}{l}\text { Started at } \\
22: 09: 39\end{array}$ & $\begin{array}{l}\text { Finished at } \\
22: 10: 21\end{array}$ \\
\hline
\end{tabular}

Secondly, an approximately one-day aged name server statistics from Soongsil university was used to get the response rate of the name server. The response rate (or cache hit rate) has a deep connection with how long name the server has been turned on, so we turned off and restarted the name server for the experiment. The response rate of name server will be the total queries responded by the name server to total queries from remote hosts.

Table 2 f summarizes the traced statistics of ns.ssu.ac.kr. on October 2, 2003, and it shows that response rate of name server is $88.48 \%$, so we assume the value of $p_{\mu}$, as

\footnotetext{
${ }^{1} n_{1}$ is always 1 .

${ }^{2}$ Following explanation is extracted from [5]. Some unimportant are excluded, and for detailed information, see [5]. RQ is the count of queries received from relay. RR is the count of responses received from relay. RIQ is the count of inverse queries received from relay. RNXD is the count of no such domain answers received from relay. RFwdQ is the count of queries received from relay that needed further processing before they could be answered. RFwdR is the count of
} 
Table 2. Name server statistics (ns.ssu.ac.kr)

\begin{tabular}{lllllll}
\hline $\begin{array}{l}\text { Date } \\
\text { Oct 2 2003 }\end{array}$ & $\begin{array}{l}\text { Place } \\
\text { Soongsil U. }\end{array}$ & & $\begin{array}{l}\text { Time } \\
\text { (in sec) }\end{array}$ & $\begin{array}{l}\text { since boot } \\
150269\end{array}$ & $\begin{array}{l}\text { since reset } \\
150269\end{array}$ & \\
\hline RQ & RR & RIQ & RNXD & RFwdQ & RFwdR & RDUPQ \\
2745590 & 823370 & 0 & 439968 & 0 & 710946 & 22794 \\
\hline RDUPR & RFail & RFErr & RErr & RTCP & RAXFR & RLame \\
1334 & 1875 & 0 & 237 & 39866 & 0 & 18549 \\
\hline ROPTs & SSysQ & SAns & SFwdQ & SFwdR & SDupQ & SFail \\
0 & 436914 & 2402330 & 316407 & 710946 & 81456 & 13 \\
\hline SFErr & SErr & RNotNsQ & SNaAns & SNXD & & \\
0 & 0 & 2352 & 131547 & 797155 & & \\
\hline
\end{tabular}

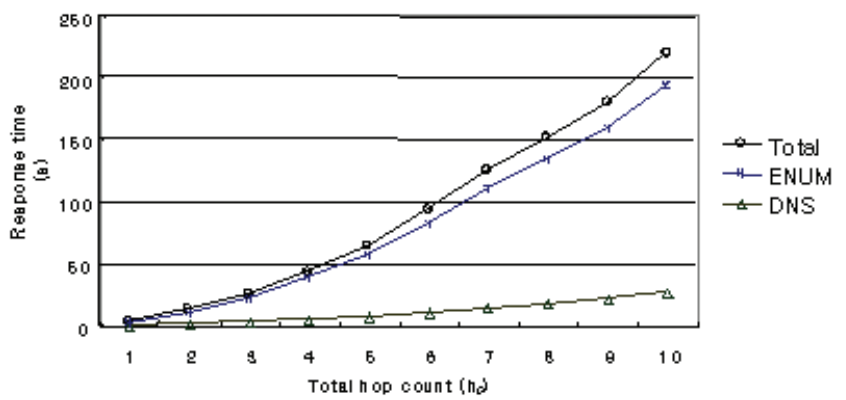

(a) $P_{\lambda}=0,1, P_{2}=0.9$

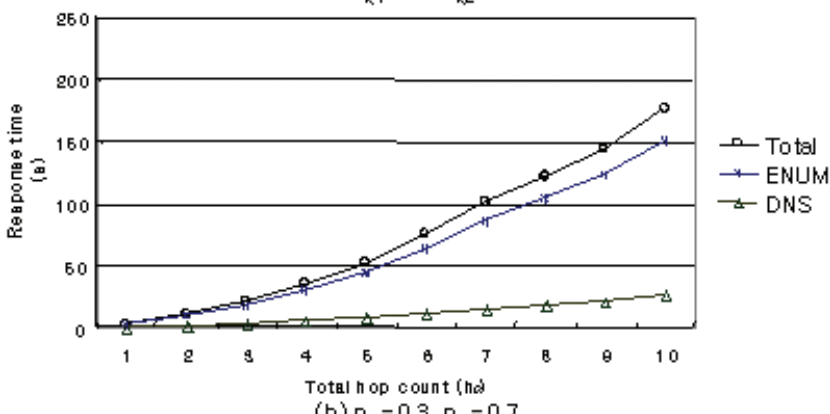

(b) $P_{2}=0.3, P_{2,2}=0.7$

Fig. 2. DNS, ENUM, and total response time

duplicate queries from relay. RDupQ is the count of duplicate queries from relay. RDupR is the count of duplicate responses from relay. RFail is the count of server failure responses from relay. SSysQ is the count of system queries sent to relay. SAns is the count of answers sent to relay. SFwdQ is the count of queries that were sent to relay when the answer was not in the name server's zone or cache. SFwdR is the count of responses from some name server that were sent to relay. SDupQ is the count of duplicate queries sent to relay. SNaAns is the count of nonauthoritative answers sent to relay. SNXD is the count of no such domains answers sent to relay. 


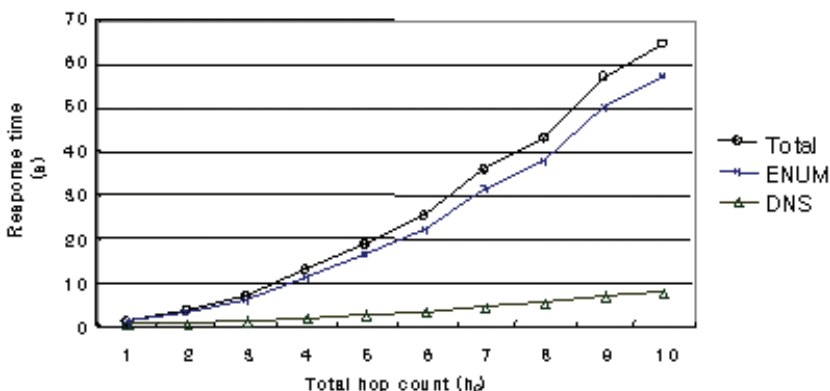

(a) $P_{2}=0,1, P_{2}=0,9$

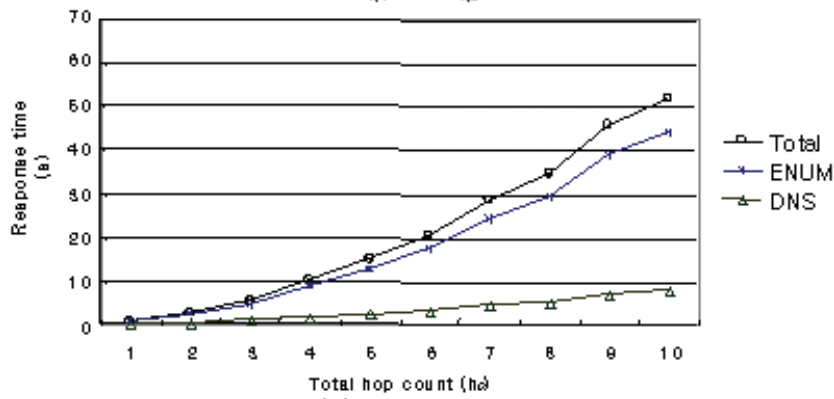

(b) $P_{\alpha}=0.3, P_{2, E}=0.7$

Fig. 3. DNS, ENUM, and total response time when NAPTR RRs are managed by area code

$p_{\mu_{1}}=0.88$, and $p_{\mu_{2}}=0.12$. In addition to it, the cache hit rate of local name server for ENUM query is supposed to be very low relatively to DNS query, and it will never get ahead of DNS's cache hit rate, because the number and size of NAPTR RR per E.164 number should be more and bigger than RR per IP, so we suspect that $p_{\mu_{1}} \ll p_{\mu_{2}}$.

The propagation time between hops is measured by transmitting 60,000 40-byte packets from one host to the first external router in SSU network on September 28, 2003. The average of propagation time, $\bar{X}$ is $43.30 \mathrm{~ms}$, and $\sigma$ is $48.98 \mathrm{~ms}$. With 95 $\%$ confidence level, the propagation time ranges from 42.91 to $43.70 \mathrm{~ms}$. The average propagation delay time between two hops is assumed to be $43.2 \mathrm{~ms}$. Propagation delay between hops follows an exponential distribution, and it is expressed as $\sigma_{i}=-\frac{\lg R}{\grave{\lambda}}$, where $R$ is random number between 0 and 1, and average propagation delay time, $\frac{1}{\bar{\lambda}}$ is $43.2 \mathrm{~ms}$.

Let us now return to formula (1). $\beta$ and $\gamma$ will be same. Router processing time, $\alpha, \beta$ and $\gamma$ are negligible compared with other variables. Formula (1) and (2) can be revised as follows, where $i \leq 1$ then $m=i$, else $m=i+1$ :

$$
\begin{gathered}
x_{i}=2 \sum_{j=1}^{m} n_{j} \delta_{j} . \\
E(X)=2 \sum \sum_{j=1}^{m}\left(n_{\lambda_{j}} p_{\lambda_{i}}+n_{\mu_{j}} p_{\mu_{i}}\right) \delta_{j} .
\end{gathered}
$$


As Fig. 2] indicate, DNS response time is insensitive to the increment of hop count and has no relevance at all to cache hit rate for ENUM. On the other hand, ENUM response time is very susceptible with increment of hop count and cache hit rate. As hop count increases from $2 h_{c}$ to $5 h_{c}$, ENUM response time at $p_{\lambda_{1}}$ rapidly jumps from $8 \mathrm{sec}$ to $44 \mathrm{sec}$ approximately. As $p_{\lambda_{1}}$ increases from 0.1 to 0.3 , ENUM response time at $5 h_{c}$ falls approximately from $57 \mathrm{sec}$ to $44 \mathrm{sec}$.

Fig. 2. proves clearly that ENUM lookup time is strongly deteriorated by increasing in the total hop count $\left(h_{c}\right)$, and total response time is decided by ENUM response time, not by DNS response time.

To improve the total response time, it is inevitable to lessen total hop count, especially caused by ENUM lookup. Name server for ENUM is bound with geographical location, so it is difficult to lesson hop count, however, if we run name server to represent each local area as proposed, query would be forwarded to directly the appropriate administrative Tier 2 name server which possess NAPTR RRs corresponding E.164 number. Fig. 3 shows that response time rapidly falls off compared with Fig. 2. Under conditions that $p_{\lambda_{1}}=0.3$ and $5 h_{c}$, ENUM response time in two experiments is roughly estimated at $44 \mathrm{sec}$ and $12 \mathrm{sec}$ respectively.

\section{Conclusions}

ENUM provides connectivity to applications based on completely different communication infrastructures, with contemporary DNS. Simply, this mechanism enables PSTN users to communicate with other parties in no matter what environment they belong to and to access to resources on Internet.

In this paper, at first, we have analyzed original DNS query response time with priority given to propagation time delay between two nodes. Secondly, we have estimated ENUM query response time with the same condition of the first analysis. Results from two experiments lead us to the conclusion that total response time is much correlated with ENUM response time. We have proposed representative Tier 2 name server, so queries from local resolvers are seldom. For the most part, queries are directly forwarded to an appropriate administrative Tier 2 name server, and we have seen this idea promises better performance in the ENUM resolution process.

\section{References}

1. Faltstrom, P.: E.164 number and DNS. RFC 2916, IETF(2000)

2. Mockapetris, P., Dunlap, K.: Development of the Domain Name System. Computer Communication Review, Vol. 18, No.4, SIGCOMM, ACM(1988) pp123-133

3. Recommendation E.164, The international public telecommunication numbering planSupplement 2: Number Portability, ITU-T (1998)

4. Next-Generation Solutions for the service-enabled Internet, third Quarter 2001 PACKET, CISCO (2001)

5. Albitz, P., Liu, C.: DNS and BIND. 4th Ed, pp185-191, O'reilly (2001).

6. Jung, J., Sit, E., Balakrishnan, H., Morris, R.: DNS Performance and the Effectiveness of Caching. Internet Measurement Workshop, SIGCOMM ACM(2001) 